\title{
Ginseng Leaf Extract Prevents High Fat Diet-Induced Hyperglycemia and Hyperlipidemia through AMPK Activation
}

\author{
Hai-Dan Yuan, Sung-Jip Kim, Hai-Yan Quan, Bo Huang, and Sung-Hyun Chung* \\ Department of Life and Nanopharmaceutical Science, Kyung Hee University College of Pharmacy, Seoul 130-701, Korea
}

This study evaluated the protective effects of ginseng leaf extract (GLE) against high fat-diet-induced hyperglycemia and hyperlipidemia, and explored the potential mechanism underlying these effects in C57BL/6J mice. The mice were randomly divided into four groups: normal control, high fat diet control (HFD), GLE-treated at $250 \mathrm{mg} / \mathrm{kg}$, and GLE-treated at $500 \mathrm{mg} /$ $\mathrm{kg}$. To induce hyperglycemic and hyperlipidemic states, mice were fed a high fat diet for 6 weeks and then administered GLE once daily for 8 weeks. At the end of the treatment, we examined the effects of GLE on plasma glucose, lipid levels, and the expression of genes related to lipogenesis, lipolysis, and gluconeogenesis. Both GLE groups lowered levels of plasma glucose, insulin, triglycerides, total cholesterol, and non-esterified fatty acids when compared to those in HFD group. Histological analysis revealed significantly fewer lipid droplets in the livers of GLE-treated mice compared with HFD mice. To elucidate the mechanism, Western blots and RT-PCR were performed using liver tissue. Compared with HFD mice, GLE-treated mice showed higher levels of phosphorylation of AMP-activated protein kinase (AMPK) and its substrate, acetyl-CoA carboxylase, but no differences in the expression of lipogenic genes such as sterol regulatory element-binding protein 1a, fatty acid synthase, sterol-CoA desaturase 1 and glycerol-3-phosphate acyltransferase. However, the expression levels of lipolysis and fatty acid uptake genes such as peroxisome proliferator-activated receptor- $\alpha$ and CD36 were increased. In addition, phosphoenolpyruvate carboxykinase gene expression was decreased. These results suggest that GLE ameliorates hyperglycemia and hyperlipidemia by inhibiting gluconeogenesis and stimulating lipolysis, respectively, via AMPK activation.

Keywords: Ginseng leaf extract, High fat diet, AMP, C57BL/6J mice

\section{INTRODUCTION}

Diabetes mellitus is a common endocrine disease characterized by hyperglycemia and long-term complications affecting the eyes, kidneys, nerves, and blood vessels [1,2]. At present, there are more than 194 million people with diabetes worldwide [3], and this number is estimated to increase to 333 million by 2025 [4]. The management of diabetes is considered a global problem, and a cure has yet been discovered. Anti-diabetic drugs such as insulin, biguanides, sulphonylureas, and alpha glucosidase inhibitors are currently used to treat diabe- tes. However, these drugs are associated with many side effects including obesity, osteoporosis sodium retention, hypoglycemia, and lactic acidosis [5-8]. To avoid such adverse side effects, many natural plants have been investigated recently as possible treatments to prevent and improve diabetes [9-11].

Ginseng, well-known medicinal plant, is widely used as an extremely valuable treatment in Oriental societies. The pharmacological effects of ginseng are attributable mainly to the ginsenosides in the plant, and many stud-

\footnotetext{
(c) This is an Open Access article distributed under the terms of the Creative Commons Attribution Non-Commercial License (http://creativecommons.org/licenses/by-nc/3.0/) which permits unrestricted non-commercial use, distribution, and reproduction in any medium, provided the original work is properly cited.
}

Received 5 Oct. 2010, Revised 27 Oct. 2010, Accepted 2 Nov. 2010

*Corresponding author

E-mail: suchung@khu.ac.kr

Tel: +82-2-961-0373, Fax: +82-2-957-0384 
ies have documented the anti-hyperglycemic and antihyperlipidemic activities of ginsenosides [12-17]. To date, more than 60 ginsenosides have been identified in different parts of the ginseng plant, and extracts of ginseng roots, rootlets, berries, and leaves have shown pharmacological activities such as islet protection, insulin sensitization, and anti-hyperglycemia, anti-obesity, and antioxidant effects [18-23].

AMP-activated protein kinase (AMPK) is a serinethreonine kinase that is activated following a rise in the intercellular AMP:ATP ratio [24]. Recently, AMPK has emerged as an attractive target molecule for the treatment of metabolic disorders, including obesity and type 2 diabetes [25]. The amelioration of hyperglycemia and hyperlipidemia by several flavonoids is thought to be mediated via AMPK activation [26,27]. However, it is not known whether the beneficial effects of the ginseng leaf on high fat diet-induced hyperglycemia and hyperlipidemia are attributable to AMPK activation. Here, we investigated the anti-hyperglycemic and antihyperlipidemic effects of ginseng leaf and the potential mechanisms underlying these actions in $\mathrm{C} 57 \mathrm{BL} / 6 \mathrm{~J}$ mice fed a high fat diet.

\section{MATERIALS AND METHODS}

\section{Preparation of ginseng leaf extract}

Ginseng leaves were dried in the shade for 1 week and extracted by boiling in $70 \%$ ethanol for $5 \mathrm{~h}$. The solution (ginseng leaf extract, GLE) was filtered and evaporated under vacuum to obtain a powder.

\section{Animals and diets}

Six-week-old C57BL/6J mice were purchased from Orient Bio (Seoul, Korea) and acclimatized to the laboratory environment for 1 week before the experiment. The mice were allowed free access to water and food and were housed under conditions of constant room temperature $\left(22 \pm 2^{\circ} \mathrm{C}\right)$ and humidity $(50 \pm 10 \%)$ with an automatic $12 \mathrm{~h}$ light: $12 \mathrm{~h}$ dark cycle. The experimental protocol was approved by the Institutional Animal Ethics Committee of Kyung Hee University. The mice were randomly divided into four groups of six mice each: normal control (Con), high fat diet (HFD), HFD+GLE at $250 \mathrm{mg} / \mathrm{kg}(\mathrm{G} 250)$, and HFD+GLE at $500 \mathrm{mg} / \mathrm{kg}$ (G500). Control mice were allowed free access to the regular diet (Con) or HFD. In the GLE-treated groups, mice were allowed free access to a HFD for 6 weeks and were then orally administered GLE once daily for 8 weeks. The composition of the experimental diet (Table
Table 1. Composition of the experimental diet

\begin{tabular}{lcc}
\hline & Regular diet & High fat diet \\
\hline Protein & $\boldsymbol{g} \%$ & $\boldsymbol{k} \boldsymbol{c} \boldsymbol{c l} \%$ \\
Carbohydrate & 24 & 20 \\
Fat & 41 & 35 \\
Kal/kg & 24 & 45 \\
Ingredient & 4,776 & \\
Casein (from milk) & $\boldsymbol{g}$ & $\boldsymbol{k c a l}$ \\
Corn starch & 200 & 800 \\
Sucrose & 155.036 & 620 \\
Dextrose & 50 & 200 \\
Cellulose & 132 & 528 \\
Soybean oil & 50 & 0 \\
Corn oil & 25 & 225 \\
Mineral mixture & 175 & 1,575 \\
Vitamin mixture & 35 & 0 \\
Tert-Butylhydroquinone (TBHQ) & 10 & 40 \\
DL-methionine & 0.014 & 0 \\
L-cystine & - & - \\
Choline bitartrate & 3 & 12 \\
Total & 2.5 & 0 \\
\hline
\end{tabular}

1) was based on the HFD $45 \%$ cal semi-synthetic diet. Body weight was measured once a week during the experiment.

\section{Determination of serum parameters}

At the end of the experimental period, blood samples were collected and the levels of plasma glucose, insulin, triglycerides (TG), total cholesterol (TC), non-esterified fatty acids (NEFA), and leptin were measured. Plasma glucose concentrations were determined using the glucose oxidase method (Asan Pharmaceutical Co., Seoul, Korea); plasma insulin concentrations, using a mouse insulin enzyme immunoassay kit (Sibayagi, Gunma, Japan); plasma TG and TC concentrations, using commercially available kits (Asan Pharmaceutical); plasma NEFA levels, using the enzymatic colorimetric method (Eiken, Tokyo, Japan); and leptin levels, using a mouse leptin enzyme immunoassay kit (Linco Research, St. Charles, MO, USA).

\section{Histological analysis}

Liver tissue was fixed in $10 \%$ neutral-buffered formalin, embedded in paraffin, and sectioned at a $5 \mu \mathrm{m}$ thickness (Leica, Wetzlar, Germany). The sections were stained with hematoxylin-eosin for microscopic assess- 
ment (Olympus, Tokyo, Japan).

\section{Western blot analysis}

After the mice were sacrificed, the liver was immediately removed, instantly soaked in liquid nitrogen, and stored at $-70^{\circ} \mathrm{C}$. Protein extracts were prepared using a protein extraction kit (Intron Biotechnology Inc., Seoul, Korea). Lysates $(40 \mu \mathrm{g})$ were electrophoresed in an $8 \%$ SDS-polyacrylamide gel, and the separated proteins were electroblotted onto a nitrocellulose membrane. The membrane was incubated for $1 \mathrm{hr}$ at room temperature in a blocking solution of Tris-buffered saline plus Tween 20 (TBST) containing 5\% skim milk (w/v), followed by incubation overnight at $4^{\circ} \mathrm{C}$ with a 1:2,000 dilution of antibody against AMPK, phospho-AMPK, acetyl-CoA carboxylase (ACC), phospho-ACC (Cell Signaling, Beverly, MO, USA), or $\beta$-actin (Santa Cruz Biotechnology, Santa Cruz, CA, USA). After four washes in $0.1 \%$ TBST, the membrane was incubated with a $1: 3,000$ dilution of horseradish peroxidase-conjugated goat antirabbit IgG or donkey anti-rabbit IgG antibody (Santa Cruz Biotechnology) for $1 \mathrm{hr}$ at room temperature. The membrane was washed four times in TBST, and immunoreactive protein bands were visualized by enhanced chemiluminescence (Amersham, Uppsala, Sweden).

\section{RNA preparation and RT-PCR}

Total mRNA was isolated from mouse livers using an Easy-Blue kit (Intron Biotechnology) according to the manufacturer's instructions. From each sample, total RNA $(10 \mu \mathrm{g})$ from each sample was reverse transcribed into cDNA using Moloney murine leukemia virus transcriptase and oligo(dT)15 primer (Promega, Madison, WI, USA). PCR was performed using the following specific sense and anti-sense primer sets: sterol regulatory element-binding protein 1a (SREBP1a), 5'-GCG CTA CCG GTC TTC TAT CA-3' and 5'-TGC TGC CAA AAG ACA AGG G-3'; fatty acid synthase (FAS), 5'-GAT CCT GGA ACG AGA ACA C-3' and 5'-AGA
CTG TGG AAC ACG GTG GT-3'; sterol-CoA desaturase 1 (SCD1), 5'-CGA GGG TTG GTT GTT GAT CTG T-3' and 5'-ATA GCA CTG TTG GCC CTG GA3'; glycerol-3-phosphate acyltransferase (GPAT), 5'GGT AGT GGA TAC TCT GTC GTC CA-3' and 5'CAT CAG CAA CAT CAT TCG GT-3'; peroxisome proliferator-activated receptor- $\alpha$ (PPAR- $\alpha), 5^{\prime}-\mathrm{CCC}$ TGA ACA TCG AGT GTC GA-3' and 5'-CTT GCC CAG AGA TTT GAG GTC CT-3'; CD36, 5'-TCCTCT GAC ATT TGC AGG TCT ATC-3' and 5'-GTG AAT CCA GTT ATG GGT TCC AC-3'; phosphoenolpyruvate carboxykinase (PEPCK), 5'-ATG CCT CCT CAG CTG CAT A-3' and 5'-TTA CAT CTG GCT GAT TCT CTG TT-3'; and cyclophilin (CPN), 5'-ATG GTC AAC CCC ACC GTG-3' and 5'-TTA GAG TTG TCC ACA GTC GGA GA-3'. CPN was amplified as a control gene. PCR was performed at $95^{\circ} \mathrm{C}$ for $5 \mathrm{~min}, 95^{\circ} \mathrm{C}$ for $30 \mathrm{sec}, 57^{\circ} \mathrm{C}$ for $30 \mathrm{sec}, 72^{\circ} \mathrm{C}$ for $30 \mathrm{sec}$, and amplified for 30 cycles. PCR was performed at of $95^{\circ} \mathrm{C}$ for $30 \mathrm{~s} ; 51^{\circ} \mathrm{C}(\mathrm{CD} 36)$, $55^{\circ} \mathrm{C}$ (SREBP1a, FAS, PPAR- $\alpha$, PEPCK, CPN), or $57^{\circ} \mathrm{C}$ (SCD1, GPAT) for $30 \mathrm{~s}$; and $72^{\circ} \mathrm{C}$ for $1 \mathrm{~min}$, with a final extension at $72^{\circ} \mathrm{C}$ for $10 \mathrm{~min}$. The PCR products were electrophoresed in a $1 \%$ agarose gel and visualized by ethidium bromide staining $(0.5 \mu \mathrm{g} / \mathrm{mL})$. Scanning densitometry was performed with an I-MAX gel image analysis system (Core-Bio, Seoul, Korea).

\section{Statistical analysis}

The results are expressed as means \pm SEM. Differences between groups were analyzed using Student's $t$-test. Statistical significance was considered for $p<0.05$.

\section{RESULTS}

\section{Effects on body weight and food intake}

Table 2 shows the effects of GLE on body weight gain, food intake, and feed efficiency in HFD-induced obese mice treated for 8 weeks. Compared with the HFD control group, the G250 and G500 groups had 3.27\% and

Table 2. Effects of ginseng leaf extract on body weight and food intake

\begin{tabular}{lccc}
\hline & Con & HFD & G250 \\
\hline Initial body weight (g) & $22.5 \pm 0.6$ & $22.2 \pm 0.3$ & $21.8 \pm 0.5$ \\
Final body weight (g) & $27.3 \pm 0.3$ & $33.6 \pm 0.8^{1)}$ & $32.5 \pm 0.5$ \\
Body weight gain (g) & $4.8 \pm 0.3$ & $11.5 \pm 0.6^{1)}$ & $31.3 \pm 0.5^{2)}$ \\
Food intake (g/mouse) & 242.1 & 241.2 & $10.7 \pm 0.7$ \\
Feed efficiency & $0.020 \pm 0.004$ & $0.048 \pm 0.006^{1)}$ & 241.2 \\
\hline
\end{tabular}

Values represent the mean \pm SE $(n=6)$

${ }^{1)} p<0.001$ vs. Con.

${ }^{2)} p<0.05$ vs. HFD. 
$6.85 \%$ lower final body weights $(p<0.05)$, respectively. The G500 group also showed lower food intake and significantly decreased feeding efficiency $(p<0.05)$ as compared with the HFD group.

\section{Effects on metabolic parameters}

Table 3 shows the effects of GLE on metabolic parameters in HFD-induced obese mice. Plasma glucose levels were significant decreased by $18.2 \%(p<0.01)$ and $22.6 \%(p<0.01)$ in the G250 and G500 groups, respectively, compared with the level in the HFD control group, and plasma insulin levels were $51.7 \%(p<0.001)$ and $52.9 \%(p<0.001)$ lower in the G250 and G500 groups as compared with the HFD control. The lower plasma glucose and insulin levels resulted in markedly lower insulin resistance index (HOMA-IR) values in the GLE-treated groups as compared with the HFD control, $60.9 \%$ lower $(p<0.001)$ in the G250 group and $63.6 \%$ lower $(p<0.001)$ in the G500 group. The plasma TG, TC, and NEFA levels were significantly lower, by $30.5 \%$ $(p<0.001), 17.8 \%(p<0.001)$, and $36.8 \%(p<0.001)$, respectively, in the G500 group as compared with the HFD group.

\section{Histological observations}

Histological analysis revealed significantly fewer lipid droplets in the livers from GLE-treated mice than in the HFD control livers (Fig. 1A).

\section{Effect on AMPK activation}

AMPK plays a key role in regulating carbohydrate and lipid metabolism and a potential therapeutic target for treatment of metabolic diseases [28]. The activation of hepatic AMPK leads to increased fatty acid oxidation and ketogenesis along with the simultaneous inhibition of hepatic fatty acids, TG, lipogenesis, cholesterol syn- thesis, and glucose production [28-31]. We examined whether GLE activates AMPK via phosphorylation in the liver. As shown in Fig. 1B, GLE-treated mice had significantly higher levels of phosphorylated AMPK and ACC than HFD control mice.

\section{Effects on gene expression related to lipogenesis, lipolysis, and gluconeogenesis}

The expression of genes involved in lipogenesis, lipolysis and gluconeogenesis were examined by RT-PCR. As shown in Fig. 1C, GLE did not affect the expressions of SREBP1a, FAS, SCD1, and GPAT, which are all associated with TG synthesis (Fig. 1C). However, the expression levels of the fatty acid transport protein CD36 gene and the lipolysis-related PPAR- $\alpha$ gene were increased in GLE-treated mice. In addition, the expression of PEPCK, a rate-limiting enzyme of the gluconeogenesis pathway, was decreased in GLE-treated mice as compared with the HFD control mice.

\section{DISCUSSION}

Ginseng is a well-known medicinal plant that has been used in traditional oriental medicine for several thousand years. In traditional Chinese medicine, ginseng root has been used more often than other plant parts; however, it is quite costly. Other parts of the ginseng plant such as berries and leaves could be harvested along with the root to yield additional herbal material and to improve the cost-effectiveness of ginseng cultivation [32]. However, one issue of concern regarding the use of ginseng as a therapeutic agent is its marginal anti-diabetic effects compared with commercially available oral hypoglycemics. To develop a more efficacious anti-diabetic agent from ginseng, GLE was considered. GLE has recently been reported to possess hypoglycemic activity [32,33],

Table 3. Effects of ginseng leaf extract on metabolic parameters in blood

\begin{tabular}{|c|c|c|c|c|}
\hline & Con & HFD & $\mathrm{G} 250$ & G500 \\
\hline Blood glucose (mM) & $15.7 \pm 0.5$ & $21.5 \pm 0.5^{1)}$ & $19.0 \pm 0.9^{2)}$ & $17.7 \pm 0.5^{1)}$ \\
\hline Insulin $(\mathrm{U} / \mathrm{mL})$ & $60.2 \pm 12.3$ & $235.6 \pm 34.1^{1)}$ & $113.9 \pm 14.1^{1)}$ & $110.9 \pm 13.8^{1)}$ \\
\hline HOMA-IR & $42.3 \pm 8.9$ & $223.7 \pm 30.8^{1)}$ & $98.2 \pm 16.2^{1)}$ & $87.5 \pm 10.9^{1)}$ \\
\hline Triglycerides (mg/dL) & $50.1 \pm 3.3$ & $87.1 \pm 3.7^{1)}$ & $67.3 \pm 3.5^{2)}$ & $60.5 \pm 2.5^{1)}$ \\
\hline Cholesterol (mg/dL) & $120.3 \pm 4.7$ & $188.2 \pm 2.7^{1)}$ & $161.3 \pm 4.1^{1)}$ & $154.7 \pm 2.5^{1)}$ \\
\hline Leptin (ng/mL) & $2.1 \pm 0.3$ & $13.7 \pm 1.6^{1)}$ & $13.8 \pm 0.9$ & $11.4 \pm 1.1$ \\
\hline $\operatorname{NEFA}(\mu \mathrm{Eq} / \mathrm{L})$ & $1151.2 \pm 63.0$ & $1700.2 \pm 62.9^{1)}$ & $1048.8 \pm 46.4^{1)}$ & $1074.0 \pm 61.2^{1)}$ \\
\hline
\end{tabular}

Values represent the mean \pm SE $(n=6)$. Plasma parameters were analyzed in plasma samples obtained from blood of 12 hr fasted mice. Homeostasis Model Assessment was used to calculate an index of insulin resistance as insulin $(\mathrm{U} / \mathrm{mL}) \times \mathrm{glucose}(\mathrm{mM}) / 22.5$.

${ }^{1)} p<0.01, p<0.001 \mathrm{vs}$. Con.

${ }^{2)} p<0.05, p<0.01, p<0.001 v s$. HFD. 
A
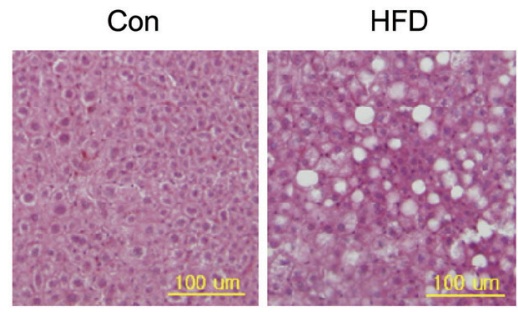

G250

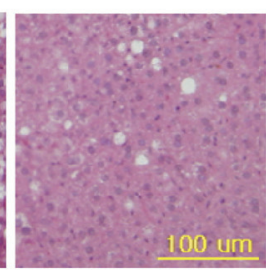

G500

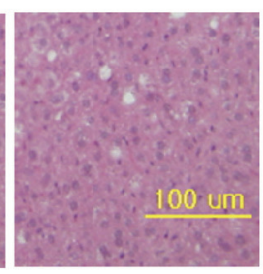

B
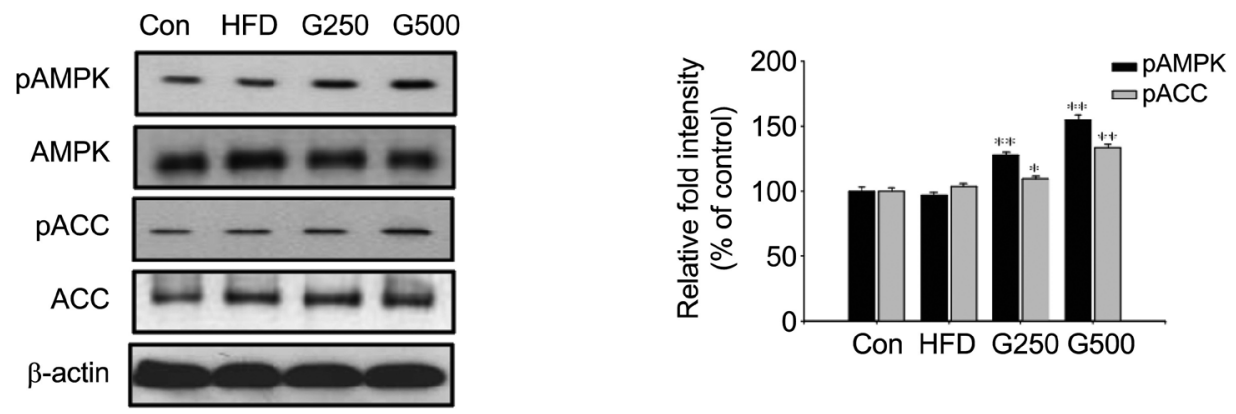

C

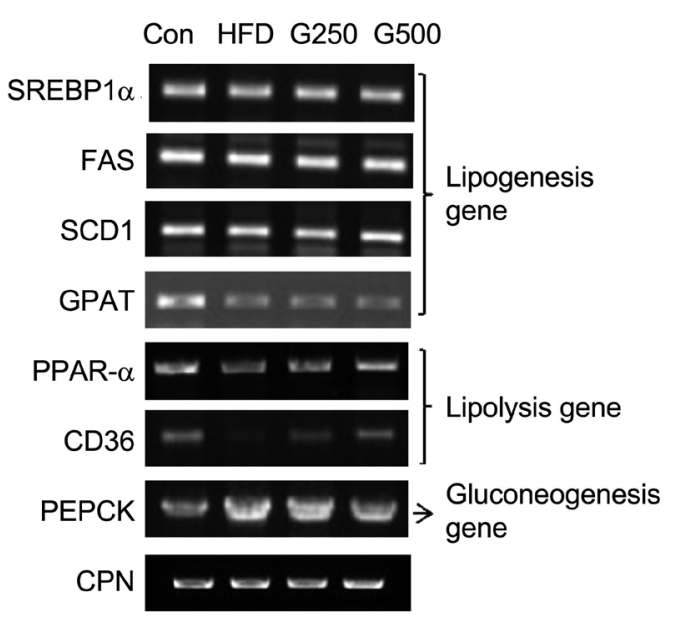

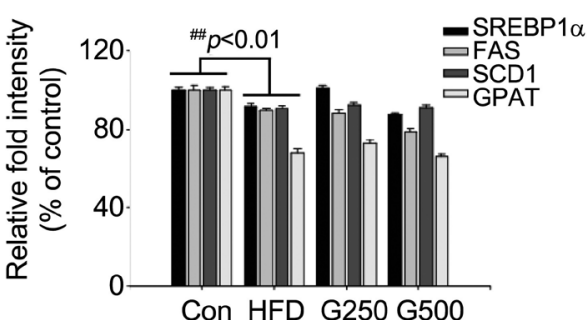

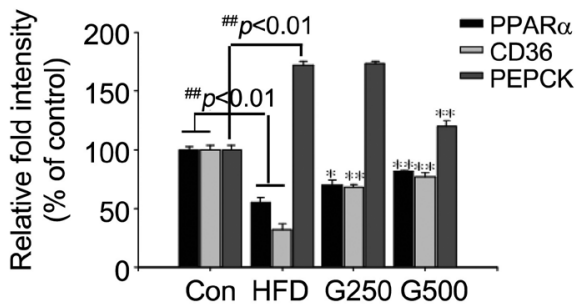

Fig. 1. Effects of GLE on liver morphology (A), AMPK and ACC phosphorylation (B) and gene expressions associated with lipogenesis, lipolysis and gluconeogenesis $C C$ ). $p<0.01$ vs. control group; $p<0.05, p<0.01$ vs. HFD control group.

although the mechanisms underlying its beneficial effects on high fat diet-induced diabetes have not been identified. In the present study, we investigated the protective effects of GLE against HFD-induced hyperglycemia in $\mathrm{C} 57 \mathrm{Bl} / 6 \mathrm{~J}$ mice and found that GLE prevented HFD-induced diabetes via AMPK activation.

Obesity is a well-recognized risk factor for type 2 diabetes, especially when combined with other known risk factors, and reduction of the risk for type 2 diabetes through weight management has been an important therapeutic goal. In the present study, GLE prevented body weight gain and significantly decreased plasma glucose and insulin levels in HFD-induced obese mice. As a result of decreased plasma glucose and insulin levels, the HOMA-IR values in the GLE groups were also signifi- cantly decreased as compared with the value in the HFD group. In addition, the levels of plasma TG, TC, NEFA, and leptin were all significantly lower in the GLEtreated group than in the HFD group. These results suggest that GLE may have beneficial effects on improving insulin resistance and hyperlipidemia induced by HFD. Histological analysis revealed a significantly lower number of lipid droplets in the livers from GLE-treated mice as compared with HFD mice (Fig. 1A). Taken together, these results demonstrate that GLE has strong effects on obesity-related hyperglycemia and hyperlipidemia.

AMPK is a heterotrimeric serine/threonine kinase that is widely expressed in a variety of organs, including the liver. Numerous studies have described the effects of AMPK activation on liver metabolism [34,35]. Two of 
the classical therapeutic targets of the system are ACC and 3-hydroxy-3-methylglutaryl-CoA reductase, which catalyze the key regulatory steps in fatty acid and sterol synthesis, respectively. The overall effects of AMPK activation in the liver include decreases in fatty acid, triglyceride, and sterol synthesis, and increases in fatty acid oxidation and ketogenesis [29-31]. In the present study, higher levels of phosphorylated AMPK and ACC were seen in GLE-treated mice as compared with HFD control mice. This result may elucidate the mechanism by which GLE promotes fatty acid oxidation and inhibits TG accumulation, thus enhancing insulin sensitivity.

It has been reported that AMPK improves a decreased ATP:AMP ratio [24]. When AMPK is activated, it inhibits lipogenic enzymes through the inhibition of SREBP1 transcriptional activity [36]. Numerous studies have indicated that SREBP1s play a key role in triglyceride synthesis by regulating lipogenesis genes such as FAS, SCD1, and GPAT. Moreover, lipolysis genes such as PPAR- $\alpha$ and CD36 also participate fatty acid oxidation [30,37]. In the present study, GLE did not affect the expression of SREBP $1 \alpha$, FAS, SCD1, and GPAT, which are all associated with TG synthesis, whereas it increased the expression of the fatty acid uptake- lipolysis-related genes CD36 and PPAR- $\alpha$. Interestingly, the expression of PEPCK, a rate-limiting enzyme in the gluconeogenesis pathway, was markedly decreased in GEL-treated mice as compared with the HFD control mice. Based on these results, we conclude that GLE stimulated fatty acid $\beta$-oxidation via AMPK activation and ACC inactivation, and increased the expression of genes associated with lipolysis (i.e., CD36, PPAR- $\alpha$ ), thereby stimulating fatty acid oxidation and decreased triglyceride content in the liver. Furthermore, through AMPK activation, GLE reduced hepatic glucose production by inhibiting PEPCK expression. As a result, GLE intercepted triglyceride amassment and ameliorated the insulin resistant status, subsequently decreasing fasting glucose levels.

In summary, we conclude that GLE prevents HFDinduced hyperglycemia and hyperlipidemia via AMPK activation in C57BL/6J mice and that GLE may be a potential therapeutic agent for type 2 diabetes or dyslipidemia.

\section{ACKNOWLEDGEMENTS}

This work was supported by a grant from the Kyung Hee University post-doctoral fellowship in 2010 (KHU20100424).

\section{REFERENCES}

1. Kim YM, Namkoong S, Yun YG, Hong HD, Lee YC, Ha KS, Lee H, Kwon HJ, Kwon YG, Kim YM. Water extract of Korean red ginseng stimulates angiogenesis by activating the PI3K/Akt-dependent ERK1/2 and eNOS pathways in human umbilical vein endothelial cells. Biol Pharm Bull 2007;30:1674-1679.

2. Tuttle KR, McGill JB, Haney DJ, Lin TE, Anderson PW; PKC-DRS, PKC-DMES, and PKC-DRS 2 Study Groups. Kidney outcomes in long-term studies of ruboxistaurin for diabetic eye disease. Clin J Am Soc Nephrol 2007;2:631636.

3. Liao Z, Chen X, Wu M. Antidiabetic effect of flavones from Cirsium japonicum DC in diabetic rats. Arch Pharm Res 2010;33:353-362.

4. Zhu CF, Peng HB, Liu GQ, Zhang F, Li Y. Beneficial effects of oligopeptides from marine salmon skin in a rat model of type 2 diabetes. Nutrition 2010;26:1014-1020.

5. Hamza N, Berke B, Cheze C, Agli AN, Robinson P, Gin H, Moore N. Prevention of type 2 diabetes induced by high fat diet in the C57BL/6J mouse by two medicinal plants used in traditional treatment of diabetes in the east of Algeria. J Ethnopharmacol 2010;128:513-518.

6. Stades AM, Heikens JT, Erkelens DW, Holleman F, Hoekstra JB. Metformin and lactic acidosis: cause or coincidence? A review of case reports. J Intern Med 2004;255:179-187.

7. Chiang CK, Ho TI, Peng YS, Hsu SP, Pai MF, Yang SY, Hung KY, Wu KD. Rosiglitazone in diabetes control in hemodialysis patients with and without viral hepatitis infection: effectiveness and side effects. Diabetes Care 2007;30:3-7.

8. Kobayashi M, Iwata M, Haruta T. Clinical evaluation of pioglitazone. Nippon Rinsho 2000;58:395-400.

9. Ryu JK, Lee T, Kim DJ, Park IS, Yoon SM, Lee HS, Song SU, Suh JK. Free radical-scavenging activity of Korean red ginseng for erectile dysfunction in non-insulin-dependent diabetes mellitus rats. Urology 2005;65:611-615.

10. Adiga S, Bairy KL, Meharban A, Punita IS. Hypoglycemic effect of aqueous extract of Trichosanthes dioica in normal and diabetic rats. Int J Diabetes Dev Ctries 2010; 30:38-42.

11. Gupta S, Sharma SB, Bansal SK, Prabhu KM. Antihyperglycemic and hypolipidemic activity of aqueous extract of Cassia auriculata L. leaves in experimental diabetes. $\mathrm{J}$ Ethnopharmacol 2009;123:499-503.

12. Han GC, Ko SK, Sung JH, Chung SH. Compound K enhances insulin secretion with beneficial metabolic effects in db/db mice. J Agric Food Chem 2007;55:10641-10648.

13. Park MW, Ha J, Chung SH. 20(S)-ginsenoside $\mathrm{Rg}_{3}$ en- 
hances glucose-stimulated insulin secretion and activates AMPK. Biol Pharm Bull 2008;31:748-751.

14. Vuksan V, Sievenpiper JL. Herbal remedies in the management of diabetes: lessons learned from the study of ginseng. Nutr Metab Cardiovasc Dis 2005;15:149-160.

15. Han KL, Jung MH, Sohn JH, Hwang JK. Ginsenoside 20S-protopanaxatriol (PPT) activates peroxisome proliferator-activated receptor gamma (PPARgamma) in 3T3L1 adipocytes. Biol Pharm Bull 2006;29:110-113.

16. Trinh HT, Han SJ, Kim SW, Lee YC, Kim DH. Bifidus fermentation increases hypolipidemic and hypoglycemic effects of red ginseng. J Microbiol Biotechnol 2007;17:11271133.

17. Cho WC, Chung WS, Lee SK, Leung AW, Cheng CH, Yue KK. Ginsenoside Re of Panax ginseng possesses significant antioxidant and antihyperlipidemic efficacies in streptozotocin-induced diabetic rats. Eur J Pharmacol 2006;550:173-179.

18. Luo JZ, Luo L. Ginseng on hyperglycemia: effects and mechanisms. Evid Based Complement Alternat Med 2009; 6:423-427.

19. Chung SH, Choi CG, Park SH. Comparisons between white ginseng radix and rootlet for antidiabetic activity and mechanism in KKAy mice. Arch Pharm Res 2001;24:214218.

20. Attele AS, Zhou YP, Xie JT, Wu JA, Zhang L, Dey L, Pugh W, Rue PA, Polonsky KS, Yuan CS. Antidiabetic effects of Panax ginseng berry extract and the identification of an effective component. Diabetes 2002;51:1851-1858.

21. Xie JT, Wang CZ, Wang AB, Wu J, Basila D, Yuan CS. Antihyperglycemic effects of total ginsenosides from leaves and stem of Panax ginseng. Acta Pharmacol Sin 2005; 26:1104-1110.

22. Davydov VV, Molokovskiı̌ DS, Limarenko AIu. Efficacy of ginseng drugs in experimental insulin-dependent diabetes and toxic hepatitis. Patol Fiziol Eksp Ter 1990;(5):4952.

23. Lü JM, Yao Q, Chen C. Ginseng compounds: an update on their molecular mechanisms and medical applications. Curr Vasc Pharmacol 2009; 7:293-302.

24. Zhou G, Myers R, Li Y, Chen Y, Shen X, Fenyk-Melody J, Wu M, Ventre J, Doebber T, Fujii N, et al. Role of AMPactivated protein kinase in mechanism of metformin action. J Clin Invest 2001;108:1167-1174.

25. Carling D. The AMP-activated protein kinase cascade: a unifying system for energy control. Trends Biochem Sci
2004;29:18-24.

26. Baur JA, Pearson KJ, Price NL, Jamieson HA, Lerin C, Kalra A, Prabhu VV, Allard JS, Lopez-Lluch G, Lewis K, et al. Resveratrol improves health and survival of mice on a high-calorie diet. Nature 2006;444:337-342.

27. Zang M, Xu S, Maitland-Toolan KA, Zuccollo A, Hou X, Jiang B, Wierzbicki M, Verbeuren TJ, Cohen RA. Polyphenols stimulate AMP-activated protein kinase, lower lipids, and inhibit accelerated atherosclerosis in diabetic LDL receptor-deficient mice. Diabetes 2006;55:21802191.

28. Winder WW, Hardie DG. AMP-activated protein kinase, a metabolic master switch: possible roles in type 2 diabetes. Am J Physiol 1999;277(1 Pt 1):E1-E10.

29. Yin HQ, Kim M, Kim JH, Kong G, Kang KS, Kim HL, Yoon BI, Lee MO, Lee BH. Differential gene expression and lipid metabolism in fatty liver induced by acute ethanol treatment in mice. Toxicol Appl Pharmacol 2007;223:225-233.

30. Horton JD, Goldstein JL, Brown MS. SREBPs: activators of the complete program of cholesterol and fatty acid synthesis in the liver. J Clin Invest 2002;109:1125-1131.

31. Hardie DG. The AMP-activated protein kinase pathway: new players upstream and downstream. J Cell Sci 2004;117(Pt 23):5479-5487.

32. Xie JT, Mehendale SR, Wang A, Han AH, Wu JA, Osinski J, Yuan CS. American ginseng leaf: ginsenoside analysis and hypoglycemic activity. Pharmacol Res 2004;49:113117.

33. Jung CH, Seog HM, Choi IW, Choi HD, Cho HY. Effects of wild ginseng (Panax ginseng C. A. Meyer) leaves on lipid peroxidation levels and antioxidant enzyme activities in streptozotocin diabetic rats. J Ethnopharmacol 2005;98:245-250.

34. Hardie DG, Carling D, Carlson M. The AMP-activated/ SNF1 protein kinase subfamily: metabolic sensors of the eukaryotic cell? Annu Rev Biochem 1998;67:821-855.

35. Hardie DG, Carling D. The AMP-activated protein kinase: fuel gauge of the mammalian cell? Eur J Biochem 1997;246:259-273.

36. Foretz M, Carling D, Guichard C, Ferré P, Foufelle F. AMP-activated protein kinase inhibits the glucose-activated expression of fatty acid synthase gene in rat hepatocytes. J Biol Chem 1998;273:14767-14771.

37. Crabb DW, Liangpunsakul S. Alcohol and lipid metabolism. J Gastroenterol Hepatol 2006;21 Suppl 3:S56-S60. 